Audiology

Neurotology
Audiol Neurotol 2010;15:343-352

DOI: $\underline{10.1159 / 000289577}$
Received: August 21, 2009

Accepted after revision: January 4, 2010

Published online: March 4, 2010

\title{
A New Model for Utricular Function Testing Using a Sinusoidal Translation Profile during Unilateral Centrifugation
}

\author{
K.I. Buytaert ${ }^{a, b} \quad$ S.A.E. Nooij ${ }^{d} \quad$ X. Neyt ${ }^{c} \quad$ P.-F. Migeotte ${ }^{c} \quad$ R. Vanspauwen ${ }^{a}$ \\ P.H. Van de Heyning ${ }^{a}$ F.L. Wuyts ${ }^{a, b}$ \\ ${ }^{a}$ Antwerp University Research centre for Equilibrium and Aerospace (AUREA), ENT Department, \\ Antwerp University Hospital, University of Antwerp, ${ }^{b}$ Laboratory of Biomedical Physics (BIMEF), \\ University of Antwerp, Antwerp, and 'Signal and Image Centre, Electrical Engineering Department, \\ Royal Military Academy, Brussels, Belgium; ${ }^{\mathrm{d}}$ TNO Human Factors, Soesterberg, The Netherlands
}

\section{Key Words}

Utricular function test - Unilateral centrifugation •

Physiological model

\begin{abstract}
The utricle plays an important role in orientation with respect to gravity. The unilateral centrifugation test allows a side-by-side investigation of both utricles. During this test, the subject is rotated about an earth-vertical axis at high rotation speeds (e.g. $400 \%$ s) and translated along an interaural axis to consecutively align the axis of rotation with the left and the right utricle. A simple sinusoidal translation profile $(0.013 \mathrm{~Hz}$; amplitude $=4 \mathrm{~cm})$ was chosen. The combined rotation and translation induces ocular counter rolling (OCR), which is measured using 3-D video-oculography. This OCR is the sum of the reflexes generated by both the semicircular canals and the utricles. In this paper, we present a new physiological model that decomposes this total OCR into a canal and a utricular contribution, modelled by a second-order transfer function and a combination of 2 sine functions, respectively. This model yields parameters such as canal gain, cupular and adaptation time constants and a velocity storage component for the canals. Utricular gain, bias, phase and
\end{abstract}

the asymmetry between the left and the right utricle are characteristic parameters generated by the model for the utricles. The model is presented along with the results of 10 healthy subjects and 2 patients with a unilateral vestibular loss due to acoustic neuroma surgery to illustrate the effectiveness of the model.

Copyright $\odot 2010$ S. Karger AG, Basel

\section{Introduction}

Patients with vestibular problems represent a relatively large group in our society (7.4\% lifetime prevalence of vertigo [Neuhauser et al., 2005]). A proper therapy is based on a correct diagnosis, which in turn is based on history taking, imaging methods as well as functional testing of the vestibular system. During the past century, the laboratory evaluation of the human vestibular system has considerably evolved. Whereas in the beginning, only the horizontal semicircular canals could be evaluated side by side using caloric testing, the clinician now has access to all separate organs using an extended battery of tests including vestibulo-ocular reflex (VOR) testing by the use of motorized rotation chairs, cervical vestibular

\section{KARGER}

Fax +41613061234 E-Mail karger@karger.ch www.karger.com
(C) 2010 S. Karger AG, Base

$1420-3030 / 10 / 0156-0343 \$ 26.00 / 0$

Accessible online at:

www.karger.com/aud
K.I. Buytaert or F.L. Wuyts

AUREA, ENT Department, Antwerp University Hospital, University of Antwerp Wilrijkstraat 10

BE-2650 Edegem, Antwerp (Belgium)

Tel.+32 382140 86, E-Mail kristof.buytaert@ua.ac.be or floris.wuyts@ua.ac.be 


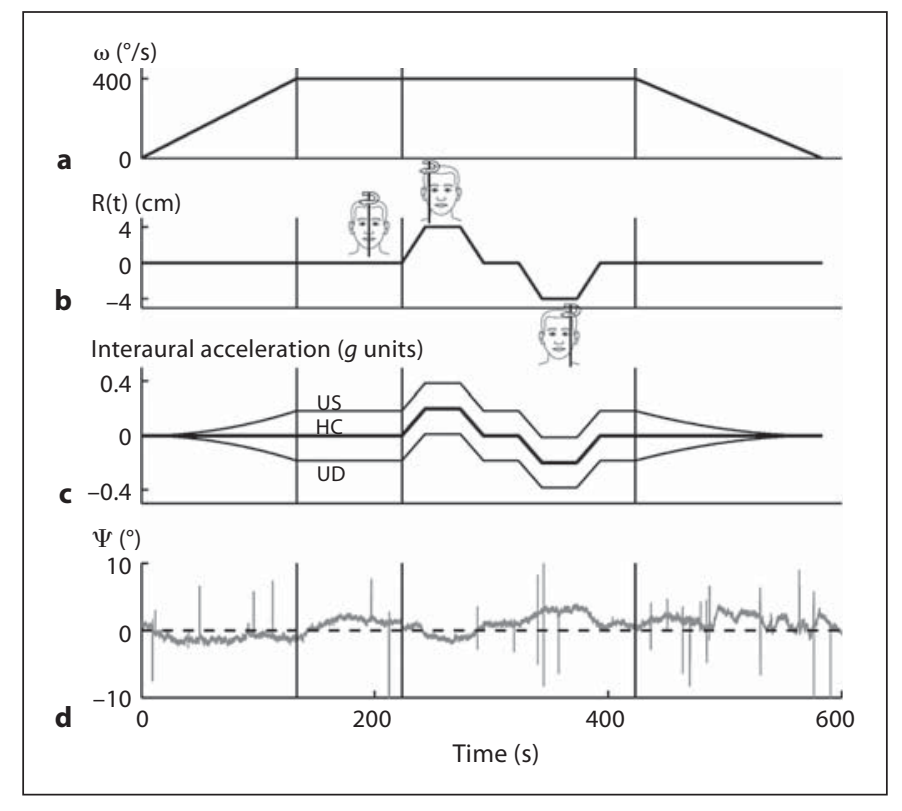

Fig. 1. Unilateral centrifugation using a trapezoidal translation paradigm. The first vertical line delineates the end of the acceleration $\left(3 \% \mathrm{~s}^{2}\right)$. The interval between the 2 first vertical lines corresponds to the steady state phase of $90 \mathrm{~s}$. The second vertical line indicates the start of the translation, and the last vertical line marks the start of the deceleration $\left(-2.5^{\circ} / \mathrm{s}^{2}\right)$. a Angular velocity $(\omega)$ profile. b Interaural translation profile. c Centripetal acceleration (in $g$ units) at the left utricle (US), the right utricle (UD) and the centre of the head (HC). $\mathbf{d}$ Example of an OCR response $(\Psi)$. Vertical spikes on the trace are due to eye blinks.

evoked myogenic potentials for predominantly saccular function evaluation and the subjective visual vertical test for the otolith function. Advanced methods include unilateral centrifugation, galvanic stimulation, ocular vestibular evoked myogenic potentials, video head impulse tests and others. The review by Wuyts et al. [2007] provides an overview of most of those techniques.

In the past two decades, technology advancement has enabled the detailed exploration of the otolith system with the elaboration of the unilateral centrifugation test [Clarke et al., 1996; Clarke and Engelhorn, 1998; Wuyts et al., 2003] as initially conceived by Yegorov and Samarin [1970], von Baumgarten and Thümler [1979] and Wetzig et al. [1990]. Throughout the past years, we have built up extensive experience with the unilateral centrifugation test [Wuyts et al., 2003] both in healthy subjects and patients with specific (vestibular) lesions. In that test, subjects are rotated about an earth-vertical axis at a velocity of $400 \%$ s. After rotating at a constant velocity for several seconds, the subject is gradually $(0.2 \mathrm{~cm} / \mathrm{s})$ translated
$4 \mathrm{~cm}$ to the right (and kept at that position for several seconds), and afterwards $4 \mathrm{~cm}$ to the left along an interaural axis. This will be referred to as the trapezoidal translation profile (fig. 1). As MRI studies have shown, the interutricular distance is almost $8 \mathrm{~cm}$, and both utricles are placed symmetrically around the centre of the head (the midpoint of the line joining the two labyrinths) [Nowé et al., 2003]. Therefore, the axis of rotation will cross one of the two utricles at certain moments during the translation. At these points, the contralateral utricle is exposed to the combination of the gravitational acceleration and a centrifugal acceleration (the vector sum of both is called the gravito-inertial acceleration) of $0.4 \mathrm{~g}$ (with $\mathrm{g}$ the gravitational acceleration which equals $9.81 \mathrm{~m} / \mathrm{s}^{2}$ ), corresponding to an apparent roll tilt of $21.7^{\circ}$, while the ipsilateral utricle is only exposed to gravity. The combined rotation and translation induces ocular counter rolling (OCR), i.e. ocular torsion, which is measured using 3-D video-oculography. The amount of OCR is a linear function of the apparent gravito-inertial tilt of the head during the lateral translation, as was shown by von Baumgarten and Thümler [1979], Wetzig et al. [1990], Diamond and Markham [1998], Clarke et al. [1999], Kondrachuk [2003] and Wuyts et al. [2003]. Using this method, the utricular sensitivity and the preponderance of the right and left utricles can be assessed separately. In most cases, the measured OCR is indeed a linear function of the gravitoinertial tilt, and relevant values for the sensitivity (slope) and preponderance (intercept) can be obtained. However, in several cases a drift can be observed during the 'plateau phases' where the axis of rotation is aligned with one of the utricles as illustrated in fig. 2. This drift makes the calculation of the linear regression less reliable.

To explain the presence of this drift, contributions of the semicircular canals were also included in the model. Thus, the unilateral-centrifugation-generated OCR was modelled by a sum of contributions of both the semicircular canals and the utricles. We applied a methodology similar to that of the analysis of the VOR that is generated by the semicircular canals during earth-vertical rotation, as developed in the seventies, e.g. by Wilson and Melville-Jones [1979]. The essence of that approach is that the semicircular canals are modelled by a second-order transfer function, characterized by parameters such as time constants, gain and phase. The most frequently used and elegant stimuli for this type of systems are sine waves. This approach has ever since been used by most of the clinical labs where patients with vertigo are investigated [Wuyts et al., 2001]. Inspired by the methodology, we therefore adopted that approach and extended it to the 


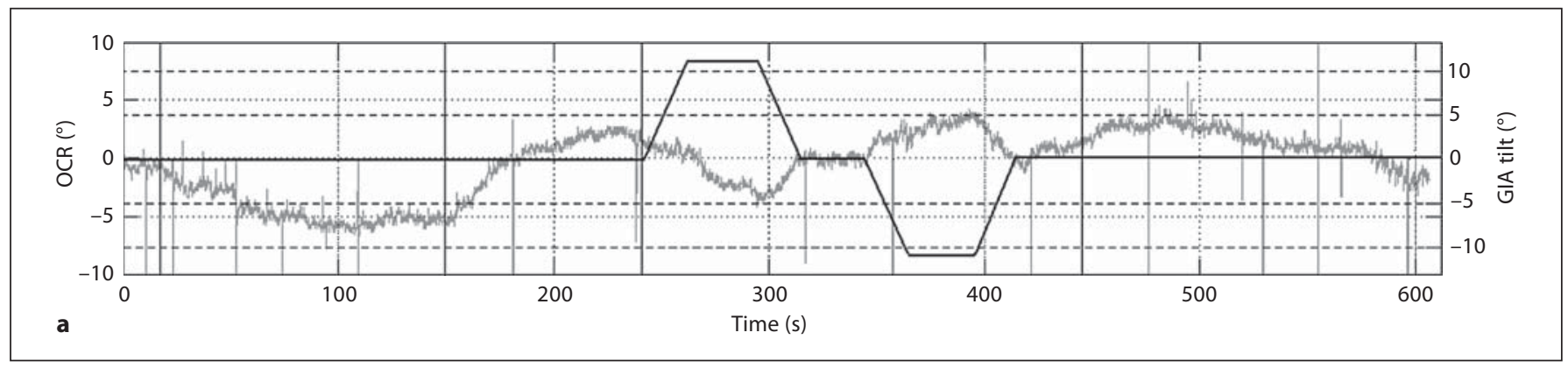

Fig. 2. a Tilt of the gravito-intertial acceleration (GIA) vector (scale on the right side of the graph) and the evoked OCR (axis on the left side of the graph) during unilateral centrifugation using the trapezoidal translation profile. Vertical spikes on the trace are due to eye blinks. In this case, a clear 'drift' is present during the acceleration and plateau phases, causing a clear non-linear behaviour when we plot the OCR as a function of the tilt of the GIA vector (b).

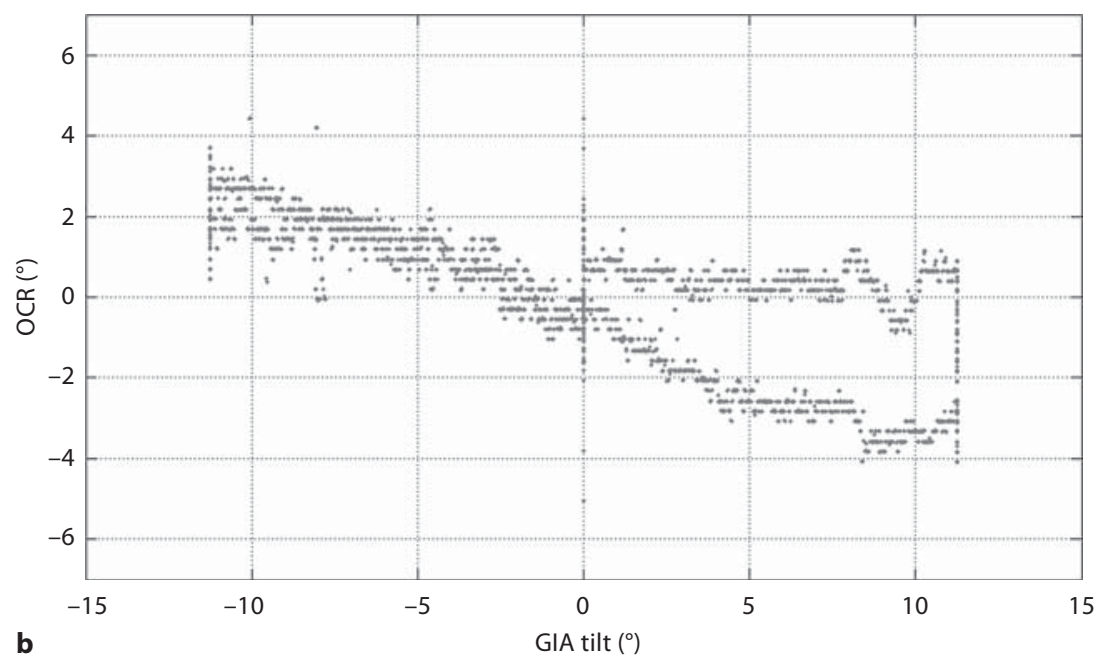

utricles. Instead of imposing a trapezoidal profile to stimulate the utricles, the subjects are repeatedly translated sinusoidally from left to right along an interaural axis.

In this paper, we present a new physiological model for the OCR induced by unilateral centrifugation and generated by both semicircular canals and utricles. We show the applicability of this new model to the sinusoidal translation pattern, yielding additional parameters that describe physiological characteristics of the semicircular canals and both utricles. We also show the added value of this modification to the trapezoid-based unilateral centrifugation test.

\section{Methods and Materials}

Subjects

Ten ( 4 male and 6 female) healthy subjects and 2 patients with a unilateral vestibular deafferentation ( $\mathrm{uVD} ; 1$ left $\mathrm{uVD}$ and 1 right $\mathrm{uVD}$ ) due to acoustic neuroma surgery were tested in this study. The data of the uVD patients were shown to illustrate the validity of the model. The study was approved by the institutional review board of the Antwerp University Hospital, and all subjects gave their written informed consent.

\section{Test Protocol}

During the unilateral centrifugation, the subject was seated in a chair rotating about the vertical axis (Neurokinetics Inc., Pittsburgh, Pa., USA) and securely fastened with a 5-point belt. The head was stabilized with a headrest and 3 flexible arms (Mitutoyo, Aurora, Ill., USA) that could be fixated against the forehead. Video-oculography was used to record and analyse 3-D eye movements, yielding horizontal, vertical and torsional coordinates in real time at a rate of $50 \mathrm{~Hz}$. Horizontal and vertical coordinates of both eyes were measured tracking a circle fitted to the pupil. The amount of OCR was calculated by means of crosscorrelation of the grey level information contained in a specific segment of the iris. A more detailed description of the video goggles and the OCR detection can be found in Kingma et al. [1995, 1997]. All measurements were performed in total darkness except for a chair-fixed faint fixation LED presented at a distance of $1 \mathrm{~m}$. That fixation LED was used to suppress horizontal nystagmus and keep the eyes close to the primary position. Prior to the rotation, the video system was calibrated using 5 fixation points that were presented under predetermined, fixed angles $\left(0^{\circ}\right.$, 


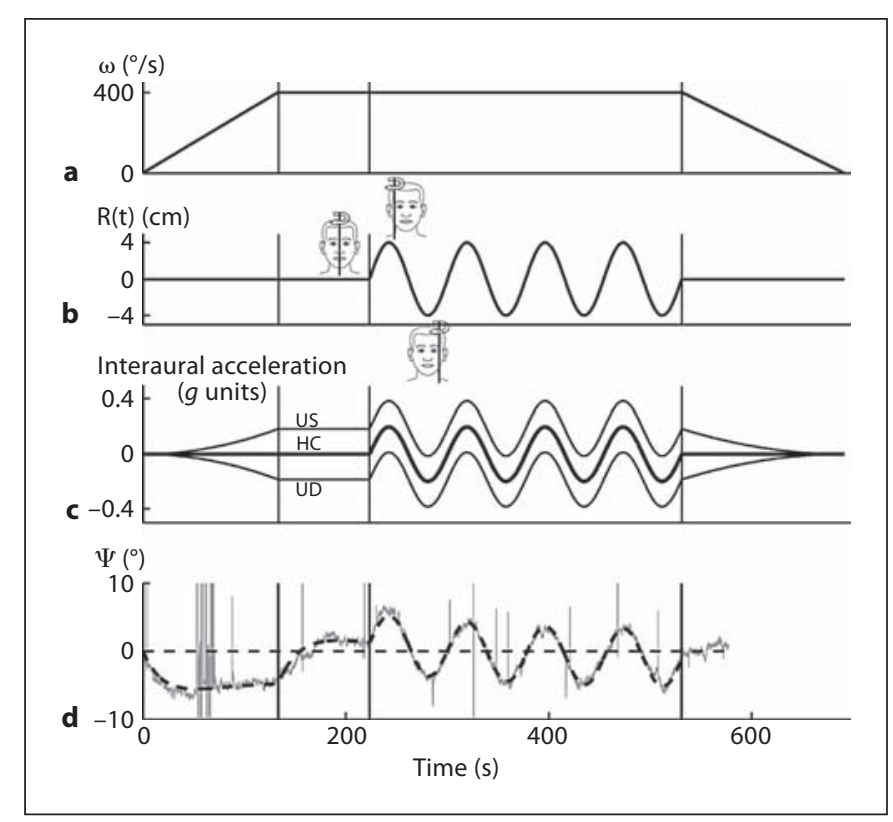

Fig. 3. Unilateral centrifugation using a sinusoidal translation paradigm. The first vertical line delineates the end of the acceleration $\left(3 \% \mathrm{~s}^{2}\right)$. The interval between the 2 first vertical lines corresponds to the steady state phase of $90 \mathrm{~s}$. The second vertical line indicates the start of the translation. The last vertical line marks the start of the deceleration $\left(-2.5^{\circ} / \mathrm{s}^{2}\right)$. a Angular velocity $(\omega)$ profile. b Interaural translation profile. c Centripetal acceleration (in $g$ units) at the left utricle (US), the right utricle (UD) and the centre of the head $(\mathrm{HC})$. d Example of an OCR response $(\Psi)$. Note that the start $(\mathrm{t}=0 \mathrm{~s})$ and stop $(\mathrm{t}=133.3 \mathrm{~s})$ of the angular yaw acceleration of the chair elicit an OCR response that is still present at the start of the translation $(t=223.3 \mathrm{~s})$. The fit of the model described by equations $1-5$ (see main text) is overlaid. The response that is recorded after the sinusoidal translation profile is not taken into account. Vertical spikes on the trace are due to eye blinks.

and $10^{\circ}$ left, right, up and down). A 30 -second baseline was recorded as a reference. At the beginning of the trial, the axis of rotation was aligned with the centre of the head, and the chair was accelerated with $3 \% \mathrm{~s}^{2}$ to a constant velocity of $400 \%$ s. Then, after a period of $90 \mathrm{~s}$ at this velocity, the chair was translated sinusoidally along the interaural axis at a frequency of $0.013 \mathrm{~Hz}$. The maximum displacement was $4 \mathrm{~cm}$ to either side. Measured at the centre of the head, this induced a maximum interaural acceleration $\left(a_{c}=\omega^{2} r\right.$, with $\omega$ the angular velocity of the chair, and $r$ the distance to the axis of rotation) of $1.95 \mathrm{~m} / \mathrm{s}^{2}(=0.2 \mathrm{~g})$, which is equivalent to a tilt of the gravito-inertial acceleration (GIA) of $11.24^{\circ}(=\arctan [0.2])$ at the centre of the head. An inclinometer (Gravitational Inclinometer ADS-100A; Watson Ind Ltd., Andover, UK) placed at the centre of the chair was used to indicate the tilt of the GIA at the centre of the head. At the maximum distance, the contralateral utricle was subjected to a centripetal acceleration of $0.4 \mathrm{~g}$, and the subject was exposed to an apparent tilt of $21.7^{\circ}(=\arctan [0.4])$.
Due to the chair translation, a very small linear Coriolis acceleration was generated. That Coriolis acceleration is given by $\alpha_{c o r}=2 v \omega$, where $v$ represents the velocity of the linear displacement $(0.002 \mathrm{~m} / \mathrm{s})$, and $\omega$ the angular velocity of the chair. The maximum Coriolis acceleration of $0.0029 \mathrm{~g}$ that could be detected was considered to be negligible.

After 4 cycles (equivalent to $307.7 \mathrm{~s}$ ) the axis of rotation was again aligned with the centre of the head, and the chair was decelerated $\left(-2.5^{\circ} / \mathrm{s}^{2}\right)$. The protocol is depicted in figure $3 \mathrm{a}-\mathrm{c}$, together with an example of the OCR response (fig. 3d).

\section{Modelling of Ocular Response during Unilateral \\ Centrifugation}

The ocular torsional position of each eye was fitted to a physiologically based mathematical model to determine the level of utricular asymmetry. In the following part, only the response of one eye will be described. Figure 3 clearly shows that both the angular acceleration of the chair (fig. 3a) and the lateral translation (fig. 3b) induce an OCR response (fig. 3d). While the part of the response induced by the lateral translation can be attributed to the utricles [Clarke et al., 1996], the angular-acceleration-induced component is most likely linked to the semicircular canals, according to Smith et al. [1995]. In order to isolate the utricular response, it is necessary to include the angular-acceleration-induced contribution in the model because it has not faded out completely at the start of translation. Thus, the total OCR response $(\Psi)$ can be described by [Nooij, 2008]:

$$
\Psi=\Psi_{S C C}+\Psi_{U}
$$

where $\Psi_{S C C}$ represents the semicircular canal contribution (SCC) related to the yaw angular acceleration, and $\Psi_{U}$ the utricular contribution that is related to the centripetal acceleration. To investigate the dynamics of the component $\Psi_{S C C}, 5$ trials of the OCR were recorded using the velocity profile of figure $3 \mathrm{a}$, but without the translation (fig. 4). When the left and right utricles are equally sensitive to centripetal acceleration, the net utricular contribution equals 0 (fig. $3 \mathrm{c}$ ), leaving only the semicircular canals to contribute to the OCR. The OCR response showed characteristics similar to those of the slow phase velocity of horizontal nystagmus during vertical axis acceleration. It contained a velocity storage component [Raphan et al., 1979] that prolonged the effective time constant of the cupular response and an adaptation component, accounting for the gradual decay during the acceleration [Oman and Young, 1972; Malcolm and Melville-Jones, 1970]. Combining those properties with a first-order model of the cupular dynamics yielded the following transfer function (Laplace notation; see also Robinson [1981] and Furman et al. [1989]):

$$
\frac{\Psi_{S C C}(s)}{\omega_{\text {head }}(s)}=A_{S C C} \times \frac{1}{1-k} \times \frac{\tau_{a} s}{\tau_{a} s+1} \times \frac{\tau_{c} s}{\left(\tau_{c} /(1-k)\right) s+1}
$$

where $A_{S C C}$ is a scaling factor, $\tau_{a}$ the adaptation time constant and $\tau_{c}$ the cupular time constant. The factor $k$ assumes a value between 0 and 1 and accounts for velocity storage. The velocity storage time constant, $\tau_{V S}$, is then given by $\tau_{V S}=\tau_{c} /(1-k)$.

Despite the intricate push-pull phenomena that govern the medial and lateral utricular partitions, as demonstrated by Uchino et al. [1999], we hypothesized in a first approximation that contributions of the utricles are linearly additive as long as the axis of rotation remains confined between or at the 2 utricles. This 
Fig. 4. Example of OCR $(\Psi)$ observed during acceleration by $3 \% \mathrm{~s}^{2}$ to $400 \%$ followed by continuous rotation during $5 \mathrm{~min}$ (300 s). The velocity profile is indicated by the dotted line (abscissa on the right). The OCR pattern shows a clear response to the start and stop of the acceleration, indicated by the vertical lines. The fit of equation 2 (see main text) is overlaid $\left(\mathrm{A}_{\mathrm{SCC}}=0.4 \mathrm{~s} ; \mathrm{k}=0.7\right.$; $\left.\tau_{\mathrm{c}}=4.4 \mathrm{~s} ; \tau_{\mathrm{a}}=173.9 \mathrm{~s}\right)$. Vertical spikes on the trace are due to eye blinks.

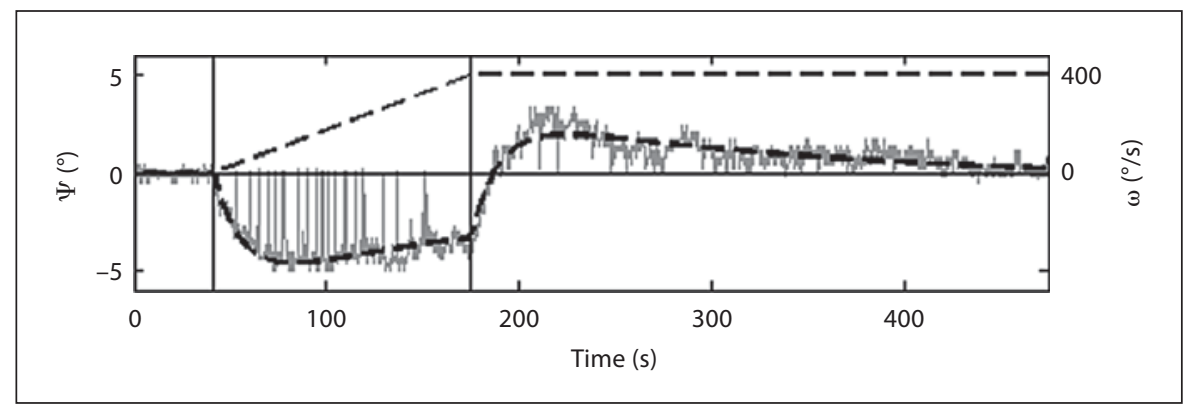

linearity was also assumed in the previous model by Wuyts et al. [2003]. Thus, the utricle-induced OCR was assumed to be proportional to the magnitude of the interaural acceleration:

$$
\begin{aligned}
& \Psi_{U}(t)=\alpha_{U S} \times \omega^{2}(t) \times\left(R_{U S}+R(t)\right) \\
& +\alpha_{U D} \times \omega^{2}(t) \times\left(R_{U D}-R(t)\right)
\end{aligned}
$$

where the first part of the right-hand side describes the contribution of the left utricle (subscript US), and the second part describes the contribution of the right utricle (subscript $U D$ ). The parameter $\alpha$ is a proportionality constant and $\omega(t)$ is the angular velocity of the chair. $R_{U S}$ is the distance between the left utricle and the centre of the head, $R_{U D}$ between the right utricle and the centre of the head. The mean interutricular distance \pm SE equals $7.45 \pm 0.08 \mathrm{~cm}$ for males and $6.99 \pm 0.06 \mathrm{~cm}$ for females, and the utricles were assumed to lie symmetrically around the centre of the head [Nowé et al., 2003]. Therefore, $R_{U D}=-R_{U S}\left(=R_{U}\right)$, and equation 3 can be simplified:

$$
\begin{aligned}
& \Psi_{U}(t)=\alpha_{U S} \times \omega^{2}(t) \times\left(R_{U}+R(t)\right) \\
& -\alpha_{U D} \times \omega^{2}(t) \times\left(R_{U}+R(t)\right)
\end{aligned}
$$

where $R(t)$ is the distance between the axis of rotation and the centre of the head. It equals 0 when the chair is on centre, and during the translation phase $R(t)$ is given by:

$$
R(t)=R_{\max } \times \sin (2 \pi f(t-d t))
$$

where $R_{\text {max }}$ is the translation amplitude $(0.04 \mathrm{~m})$ and $f$ the translation frequency $(0.013 \mathrm{~Hz})$. The term $d t$ was incorporated to account for possible phase differences between the actual translation of the chair and the ocular response. It can be positive as well as negative, similar to the VOR phase during the sinusoidal harmonic acceleration test (SHAT) with earth-vertical axis rotation.

The ocular torsion (position) data was fitted by the model described by equations 1-5 to obtain individual values for the parameters $A_{S C C}, \tau_{c}, \tau_{a}, \tau_{V S}, \alpha_{U S}, \alpha_{U D}$ and $d t$. An example of a model fit is depicted in figure $3 \mathrm{~d}$. Other examples are shown in figure 5 .

Utricular asymmetry (UA) was present when the proportionality constants $\alpha_{U D}$ and $\alpha_{U S}$ differed in magnitude, and it was defined as:

$$
U A=\frac{\alpha_{U S}-\alpha_{U D}}{\alpha_{U S}+\alpha_{U D}} \times 100 \%
$$

Since $\alpha_{U S}$ and $\alpha_{U D}$ are both positive, UA ranges between -100 and $100 \%$ (complete unilateral loss on the left or right side, respectively). In case of a perfect symmetry $\left(\alpha_{U S}=\alpha_{U D}\right)$, UA equals 0 .
Additional parameters used to characterize the response were the bias and amplitude $A_{U}$ of the total utricular response $\Psi_{U}$. Both bias and $A_{U}$ can be derived from equation 4 and are given by:

$$
\begin{aligned}
& \text { Bias }=\left(\alpha_{U S}-\alpha_{U D}\right) \times \omega^{2} \times R_{U} \\
& A_{U}=\left(\alpha_{U S}+\alpha_{U D}\right) \times \omega^{2} \times R_{\max }
\end{aligned}
$$

The utricular gain was calculated as $A_{U} / 11.24^{\circ}$ (response/stimulus at centre of head).

A Matlab procedure (version R2007a; The MathWorks Inc., Natick, Mass., USA) using the Levenberg-Marquardt algorithm was created to perform the least squares curve fit and calculate the characteristic parameters [Levenberg, 1944; Marquardt, 1963]. The fit procedure is based on an iterative procedure, as well as the knowledge of the frequency of the sine and the interutricular distance, i.e. average values are adopted for either men or women. Data points of the OCR response are removed when they are beyond 2 standard deviations of the residuals between the fit and data points for the entire curve. The fit procedure was ended after maximally 15 repetitions, or with a minimum $R^{2}$ value of 0.92 . Table 1 provides the boundaries of the parameters that were used during the fitting procedure, as well as the initial values that were given to the parameters to start that procedure. It should be noted that even in the case of a uVD ear, none of the variables were manually set to 0 .

\section{Results}

Figure 5 depicts the response of the left eye of a healthy subject, and table 2 (top row) gives an overview of the model parameters obtained from the left eyes of 10 healthy subjects. The averaged $95 \%$ CI of the fitted parameters are given in table 3 (top row). Those CI were determined during the fitting procedure and represent the uncertainty of the final value of each fitted parameter. Thanks to the Levenberg-Marquardt algorithm and the fact that outlying measurements are excluded by the fitting procedure, a robust fit is guaranteed even in the presence of artefacts, for example due to eye blinks of the subject. On average, an $\mathrm{R}^{2}$ value of 0.92 was obtained.

Negative angles represent anticlockwise torsion, positive angles clockwise OCR from the subject's point of 


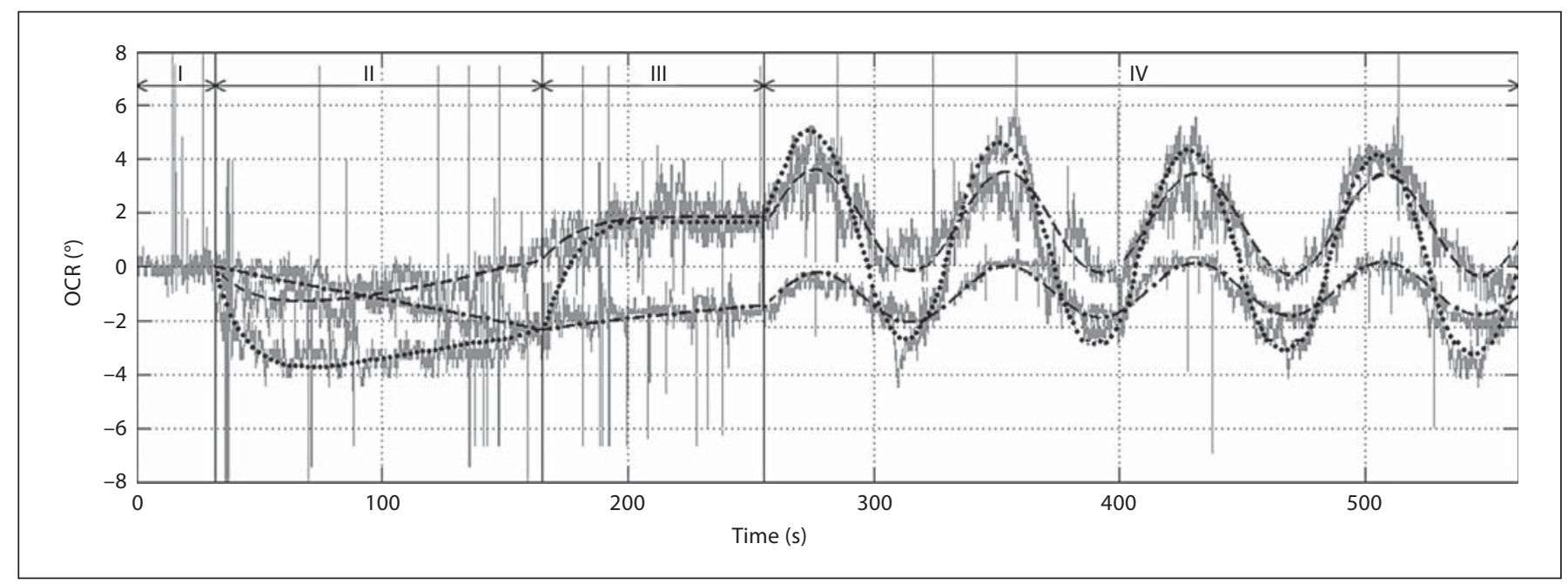

Fig. 5. Test outcome of unilateral centrifugation test in a healthy subject (dotted line), in a patient with a unilateral vestibular loss on the left side (dashed-dotted line) and in another patient with a loss on the right side (dashed line). In case of a unilateral loss, a clear shift in the (sinusoidal) profile towards the affected side is present. The fit of the model described by equations $1-5$ is overlaid. For the healthy subject, this yields the following parameters: $\mathrm{A}_{\mathrm{SCC}}=0.29 \mathrm{~s}, \tau_{\mathrm{c}}=4.24 \mathrm{~s}, \tau_{\mathrm{a}}=353 \mathrm{~s}, \tau_{\mathrm{vs}}=12.6 \mathrm{~s}, \mathrm{dt}=0.30 \mathrm{~s}, \mathrm{UA}=$
$-15.6 \%$, bias $=0.34^{\circ}$ and utricular gain $=0.300$. The parameters of the 2 patients with a unilateral loss are given in table 2 . Vertical spikes are due to eye blinks of the subject. Negative angles represent anticlockwise torsion, positive angles clockwise OCR from the subject's point of view. Part I: pre-rotation baseline. The interval between the 2 first vertical lines corresponds to the acceleration phase (part II). Part IV indicates the sinusoidal translation of the chair, after a steady state of $90 \mathrm{~s}$ (part III).
Table 1. Lower and upper boundaries and initial parameter values used during the fitting procedure

\begin{tabular}{llll}
\hline Parameter & $\begin{array}{l}\text { Lower } \\
\text { boundary }\end{array}$ & $\begin{array}{l}\text { Upper } \\
\text { boundary }\end{array}$ & $\begin{array}{l}\text { Initial } \\
\text { fit value }\end{array}$ \\
\hline$\tau_{\mathrm{c}}[\mathrm{s}]$ & 3 & 12 & 4.2 \\
$\tau_{\mathrm{a}}[\mathrm{s}]$ & 10 & 700 & 200 \\
$\mathrm{k}$ & 0 & 1 & 0.75 \\
$\mathrm{~A}_{\mathrm{SCC}}[\mathrm{s}]$ & -15 & 15 & 1 \\
$\alpha_{\mathrm{US}}\left[\mathrm{s}^{2} /\left({ }^{\circ} \mathrm{m}\right)\right]$ & 0 & 2 & 1 \\
$\alpha_{\mathrm{UD}}\left[\mathrm{s}^{2} /\left({ }^{\circ} \mathrm{m}\right)\right]$ & 0 & 2 & 1 \\
$\mathrm{dt}[\mathrm{s}]$ & -20 & 20 & 0 \\
\hline
\end{tabular}

view. The different phases of the protocol (baseline, acceleration, steady state and translation) are delineated with vertical markers.

Figure 5 also depicts the test outcome of 2 patients with a unilateral vestibular loss (left and right $\mathrm{uVD}$, respectively) due to an acoustic neuroma surgery. As could be expected, the traces of the uVD patients are shifted (vertically) to one side, especially during the translation phase. The direction of the shift depends on the side of the lesion. A left $\mathrm{uVD}$ results in pronounced anticlock- wise OCR, a right $\mathrm{UVD}$ in pronounced clockwise OCR. This shift ('asymmetry') can be explained as follows: before the start of the rotation, i.e. during $30 \mathrm{~s}$ of standstill (part I in fig. 5) and in full darkness, the OCR is per definition equal to 0 . This is the baseline of the entire test. In part II, the chair is accelerated at $3 \% \mathrm{~s}^{2}$, giving rise to a response from both the SCC and the utricle of the unaffected side. This stimulation induces horizontal nystagmus, which is suppressed thanks to the continuous use of a fixation light. Apart from the nystagmus, the acceleration stimulates the SCC that produces an OCR, according to the second-order transfer function as described by Furman et al. [1989] in equation 2. The acceleration also exerts a gradual centrifugal force (proportional to $\omega^{2} R$ ) on the unaffected utricle, generating an OCR as well. Both OCR responses from the SCC and the utricle are added in the model.

Then, in part III of the graph, the rotation chair is at full speed $(400 \%)$. The OCR due to the stimulation of the SCCs subsides, governed by the second-order transfer function (see above). Indeed, the canals are only stimulated by acceleration. During this subsidence of the SCC response, the net stimulus for the vestibular system is the continuous rotation and thus a centrifugal force of $0.2 \mathrm{~g}$, oppositely directed for the left and right vestibular sys- 
Table 2. Model parameters of left eye for 10 healthy subjects (HS) and 2 patients with uVD due to acoustic neuroma surgery

\begin{tabular}{llllllllllll}
\hline & $\mathrm{A}_{\mathrm{SCC}}[\mathrm{s}]$ & $\mathrm{M}_{\mathrm{SCC}}\left[{ }^{\circ}\right]$ & $\tau_{\mathrm{c}}[\mathrm{s}]$ & $\tau_{\mathrm{a}}[\mathrm{s}]$ & $\tau_{\mathrm{VS}}[\mathrm{s}]$ & $\alpha_{\mathrm{US}}\left[\mathrm{s}^{2} /\left({ }^{\circ} \mathrm{m}\right)\right]$ & $\alpha_{\mathrm{UD}}\left[\mathrm{s}^{2} /\left({ }^{\circ} \mathrm{m}\right)\right]$ & $\mathrm{dt}[\mathrm{s}]$ & UA [\%] & Bias [ $\left.{ }^{\circ}\right]$ & Utricular gain \\
\hline 10 HS & $0.20(0.11)$ & $-2.55(0.88)$ & $4.64(0.44)$ & $270(160)$ & $22(14)$ & $0.65(0.25)$ & $0.70(0.28)$ & $0.8(1.2)$ & $2(22)$ & $0.46(0.34)$ & $0.233(0.069)$ \\
Left uVD & -0.112 & -0.95 & 6.47 & 14 & 14.1 & 0 & 0.71 & 2.36 & -100 & -1.29 & 0.12 \\
Right uVD & -0.099 & -1.81 & 4.85 & 700 & 20.23 & 0.57 & 0 & 1.43 & 100 & 1.03 & 0.10 \\
\hline
\end{tabular}

Values are means (SD). $\mathrm{M}_{\mathrm{SCC}}=$ Maximal amplitude of the OCR during the acceleration phase.

Table 3. Averaged 95\% CI of fitted parameter for 10 healthy subjects (HS) and 2 patients with uVD due to acoustic neuroma surgery

\begin{tabular}{|c|c|c|c|c|c|c|}
\hline & $\mathrm{A}_{\mathrm{SCC}}[\mathrm{s}]$ & $\tau_{c}[s]$ & $\tau_{\mathrm{a}}[\mathrm{s}]$ & $\alpha_{\mathrm{US}}\left[\mathrm{s}^{2} /\left({ }^{\circ} \mathrm{m}\right)\right]$ & $\alpha_{\mathrm{UD}}\left[\mathrm{s}^{2} /\left({ }^{\circ} \mathrm{m}\right)\right]$ & dt $[s]$ \\
\hline $95 \%$ CI 10 HS & $0.145,0.255$ & $0.84,8.44$ & 182,358 & $0.648,0.652$ & $0.695,0.705$ & $0.72,0.88$ \\
\hline 95\% CI left uVD & $-0.117,-0.107$ & $5.62,7.32$ & $-75,103$ & $-0.004,0.004$ & $0.706,0.714$ & $2.27,2.45$ \\
\hline $95 \%$ CI right uVD & $-0.139,-0.059$ & $4.45,5.25$ & 625,775 & $0.56,0.58$ & $-0.009,0.009$ & $1.30,1.56$ \\
\hline
\end{tabular}

To perform the curve fit and determine the characteristic parameters, the Levenberg-Marquardt algorithm was used.

tems. In case of the 2 functional utricles, this generates OCR of opposite signs, so that they cancel each other out. However, in the case of the patient with a uVD, the unaffected utricle already generates a measurable OCR, corresponding to a stimulation of $0.2 \mathrm{~g}$. The utricle-mediated OCR is added to the OCR generated by the SCCs that is gradually subsiding. Although it looks like a baseline, it is certainly not to be considered as such. It is a line around which the subsequent sine pattern oscillates, but this line is subsiding, thus in general it is not horizontal. We defined the bias as the amplitude of this line, after compensation for the SCC-generated OCR, i.e. after the line has imaginarily been made horizontal, and is purely a utricular contribution. When the 'bias line' is relatively horizontal, the time constant $\tau_{a}$ from the SCC is relatively small. When a clear shift is observed till the end of the sinus part, a high $\tau_{a}$ is obtained.

At the beginning of part IV of the graph, the chair starts to translate to the left, so the axis of rotation moves to the right of the patient, and at the end of the first quarter of a cycle, the axis passes through the right utricle. In case of a left $\mathrm{uVD}$, this is the only functional utricle. In a healthy situation, the opposite (left) side would generate an OCR corresponding to a stimulus of $0.4 \mathrm{~g}$, but since the left vestibular system is deafferented, no utricle-mediated
OCR is generated. Therefore, this response is identical to the zero OCR that was obtained at the very beginning of the experiment before rotation, except for the canal contribution. When the chair moves to the centre position at full speed, the only functional utricle again produces its OCR. This could erroneously be seen as a baseline, but it is not. Moreover, the OCR that is observed when the chair symmetrically moves to both eccentric positions is symmetric around this 'net' OCR. In healthy subjects, this net OCR is indeed 0 , because of the cancelling out of each utricular response, but in uVD patients this is not the case. In the trapezoid approach [Wuyts et al., 2003], this net OCR was called the intercept. The shift in OCR response is also reflected in the proportionality constant $\alpha$, which is 0 for the side of the $\mathrm{uVD}$, yielding a complete asymmetry of $100 \%(-100 \%$ in the case of a left $\mathrm{uVD}$, and $100 \%$ in the case of a right $\mathrm{uVD}$ ), as illustrated in table 2 .

In addition to the vertical shift in the OCR traces of the uVD patients, the amplitude of the sine is lower than that of the healthy subjects as well, reflecting the lower utricular gain for the uVD patients compared to the healthy subjects. The gain of the latter is almost 2 times larger than that of the patients (table 2). Table 3 provides the $95 \%$ CI of the fitted parameters as obtained by the mathematical fitting procedure. 


\section{Discussion}

The subjective visual vertical test is often used to assess utricular function. However, OCR appears to be a more direct method to assess the utricular function without the confounding influence of central processing [Tarnutzer et al., 2009]. Thanks to recent advances in image analysis, the appropriate analysis of 3-D video-oculographic data has enabled a refined extraction of OCR that is evoked during the stimulation of the peripheral vestibular system. As a response to the improved analysis methods, new stimulation methods have emerged during the past decade to investigate the vestibular organs in detail [Clarke et al., 2003; Wuyts et al., 2003]. A refinement of those methods is crucial to obtain detailed unilateral characteristics. Concomitant with new analysis and stimulation paradigms, the models to interpret the test outcome need to be improved. The current paper thus offers a second-generation unilateral centrifugation paradigm where the original trapezoidal stimulation profile that has been used in previous studies [Wuyts et al., 2003] is replaced by the more robust sinusoidal profile. The whole approach is based on a physiological model of OCR generated by the utricles and the semicircular canals. The latter comprise the combined left and right canal function, similar to the angular VOR during SHAT on standard earth-vertical rotation chairs [Wolfe et al., 1978; Wall, 1990]. Similar to the previous model for the angular VOR, the current model cannot differentiate between the left and right canal functions. The SHAT model does not yield characteristics of both utricles separately, in contrast to the new model.

In addition to the previous approach of the unilateral centrifugation test using a trapezoidal translation profile where only a gain and a preponderance factor (dominance of one of the two utricles) could be calculated, the current model yields parameters for both the canals and each utricle.

Moreover, the current analysis offers a more robust and reliable approach to determine unilateral utricular characteristics for the following reasons. Firstly, it incorporates gender differences regarding the distance between the utricles, which has not been done in previous models [Clarke and Engelhorn, 1998; Wuyts et al., 2003]. Secondly, the response characteristics of the model are determined at the single frequency of the sine wave used. Since it is known that the utricule-mediated linear VOR is frequency dependent [Crane et al., 1997; Paige et al., 1998], it is preferred to determine the model parameters for a specific frequency instead of using a trapezoid paradigm, which intrinsically has a broad spectrum of frequencies. Moreover, the use of a sine has the advantage that the input-output function is quantifiable in terms of gain and phase, whereas other excitation profiles are much more complicated to parameterize. Obviously, when comparing data from different laboratories, it is recommended to mention the frequency of the sine wave as it is standard practice for earth-vertical axis rotation testing to determine the angular VOR characteristics. On the other hand, the frequency dependency of the OCR gain in this lowfrequency domain is fairly limited [Clarke et al., 1999]. Thirdly, the analysis of the raw data is based on the knowledge of the sine wave stimulation, increasing significantly the robustness of the fitting procedure. Fourthly, this approach uses the OCR response generated during the entire test procedure comprising the acceleration, steady state rotation and translation phases. This is in contrast to methods of unilateral centrifugation where only parts of the plateau phases are used to determine the outcome parameters. Finally, our approach incorporates the canal contribution, which appears to be very variable interindividually, thus it seems very difficult to neglect it. In a first approach, the canal-generated OCR could be neglected by waiting longer before starting the actual sideways translation. Figure 4 clearly shows, however, that the canal-generated OCR only subsided after $5 \mathrm{~min}$ of constant rotation in this healthy subject. In case of an affected vestibular system, the time constants may be greatly affected, and remaining effects could still be present after $5 \mathrm{~min}$ of constant rotation before the start of the translation. An increased steady state phase would lengthen the test procedure considerably, which is surely to be avoided in cases of patients with vertigo as they have to rotate at such high speeds (e.g. $400 \%$ s).

When compared to the previously published data on trapezoidal unilateral centrifugation, the utricular gain factor that was calculated for the 10 healthy subjects using the sinusoidal translation profile (average $=0.233$; $\mathrm{SD}=$ 0.069) corresponds to the gain that was found in the earlier study with the trapezoidal translation profile (average $=0.232$; $S D=0.054$ ) [Wuyts et al., 2003]. In the 2 patients with a uVD, the utricular gain was halved. Those findings corroborate the linear and additive character of the utricular response, as has already been proposed by other authors [von Baumgarten and Thümler, 1979; Wetzig et al., 1990; Diamond and Markham, 1998; Clarke et al., 1999; Kondrachuk, 2003; Wuyts et al., 2003]. The bias, the offset value of the utricular response, corresponds to the intercept of the linear regression model by Wuyts et al. [2003] and is correlated with the asymmetry. 
For clinical purposes, it has to be interpreted together with the other variables such as the utricular gain.

As expected, the proportionality constant $(\alpha)$ of the utricular contribution was 0 for the uVD side, yielding a complete asymmetry of $100 \%(-100 \%$ in case of a left $\mathrm{uVD}$, and $100 \%$ in case of a right $\mathrm{uVD}$ ). The fact that, in case of $\mathrm{uVD}$ patients, the model fitting results in a 0 for the $\alpha$ of the defected side is a validation of the model. If this would not be the case for a known uVD pathology, the presented model would not be realistic. That asymmetry was also reflected in the shift in OCR traces, especially during the translation phase. The shift in the traces depended on the side of the uVD and could be explained as follows: in case of a uVD, only the unaffected utricle is stimulated throughout the test procedure. The subject feels tilted to the unaffected side throughout the entire experiment, except in those moments that the axis of rotation is perfectly aligned with the unaffected utricle. Consequently, both eyes counter roll towards the affected side (anticlockwise in the case of a left $\mathrm{uVD}$, clockwise in the case of a right $\mathrm{uVD}$ ) to compensate for the apparent tilt, resulting in the observed shift in the OCR trace.

The fact that the average asymmetry factor of the healthy subjects differs from 0 corroborates the findings by von Baumgarten and Thümler [1979], Diamond and Markham [1992] and Peterka [1994]. They found a functional asymmetry between the left and the right utricle that might be explained by differences in size or weight. The sample size of the group presented here may be too small to generalize these findings.

Unlike the linear model that was used to interpret the test outcome of the trapezoidal translation [Wuyts et al., 2003], the current model not only allows the calculation of utricular characteristics, but it also enables the calculation of specific canal parameters. One of those parameters is the cupular time constant, which indicates how long it takes for the cupula to return to its original position after the onset of the constant angular acceleration. The reported average cupular time constant $\tau_{c}$ of $4.64 \mathrm{~s}$ $(\mathrm{SD}=0.44 \mathrm{~s})$ for the 10 subjects is very well in accordance with the results found by Dai et al. [1999], who obtained a cupular time constant of $4.2 \mathrm{~s}(\mathrm{SD}=0.6 \mathrm{~s})$. In the same study, Dai et al. [1999] found a velocity storage time constant of $16.7 \mathrm{~s}(\mathrm{SD}=5.2 \mathrm{~s})$, whereas it is $22 \mathrm{~s}(\mathrm{SD}=14 \mathrm{~s})$ in the present study. The results from the present study do not differ significantly (Wilcoxon's signed-rank test: $\mathrm{p}>$ 0.05) from those from the study by Dai et al. [1999].

It should be remarked that the data reported by Dai et al. [1999] comprise the canal characteristics based on a horizontal VOR model and not on the torsional VOR model as in our study, but it is physiologically very unlikely that the mechanical cupular time constant, determined by elastic, inertial and viscous properties, would be different for the two models.

The large intersubject differences (reflected in the relatively large SE), especially for the adaptation time constant $\tau_{a}$, could be due to a great inter-individual variability in central nervous system processing and should be further investigated. Nevertheless, the model presented here allows such a large (natural) variability.

Whereas the utricular gain accounts for the responsiveness of the utricles, the maximal canal contribution $\left(M_{S C C}\right)$ can be seen as a measure of the responsiveness of the semicircular canals. The minus sign indicates an anticlockwise torsion (from the subject's point of view). As described by Smith et al. [1995], that anticlockwise direction is due to the direction of the angular acceleration (clockwise in the present study). Despite the larger intersubject differences in $M_{S C C}$, the canal contribution is significantly smaller in the UVD patients than in the healthy subjects. It should, however, be noted that the $M_{S C C}$ is a combined output of the left and right semicircular canal systems, whereas even a hypofunction could lead to normal OCR, generated by the canals at this phase of the stimulation paradigm, i.e. on centre rotation.

The current model does neither incorporate the possible prevalence of the medial versus lateral partitions of the utricles nor any possible dominant projection from the utricles to ipsi- or contralateral eye muscles. This explains why we did not elaborate yet on a possible disconjugacy between both eyes. Nevertheless, the presence of any disconjugacy would not affect the principle of the model presented.

\section{Conclusion}

The drift as seen during the original unilateral centrifugation paradigm using a trapezoidal translation profile [Wuyts et al., 2003] has initiated the search for an alternative approach. By including the contribution of the semicircular canals, the overall OCR response obtained during the unilateral centrifugation test can be described more adequately. The sinusoidal translation profile significantly improves the robustness of the fitting procedure and yields additional parameters describing physiological characteristics of each of the two utricles. The model proves to be robust to fit different types of responses, from healthy subjects to patients with unilateral vestibular loss. The outcome parameters that can be com- 
pared with their counterparts from the literature are very much in accordance. The present model therefore appears to be very useful both in clinical and experimental settings to further exploit OCR induced by unilateral centrifugation, yielding physiological characteristics of the vestibular organ comprising the semicircular canals and both utricles.

\section{Acknowledgements}

The authors would like to thank the subjects that participated in the rotation experiments. This study was funded by the Research Foundation Flanders (fellowship: K.I.B.) and PRODEX.

\section{References}

-Clarke AH, Engelhorn A, Scherer H: Ocular counterrolling in response to asymmetric radial acceleration. Acta Otolaryngol 1996; 116:652-656

Clarke AH, Engelhorn A: Unilateral testing of utricular function. Exp Brain Res 1998;121: 457-464.

Clarke AH, Engelhorn A, Hamann C, Schönfeld $\mathrm{U}$ : Measuring the otolith-ocular response by means of unilateral radial acceleration. Ann NY Acad Sci 1999;871:387-391.

Clarke AH, Schönfeld U, Helling K: Unilateral examination of utricle and saccule function. J Vestib Res 2003;13:215-225.

-Crane BT, Viirre ES, Demer JL: The human horizontal vestibulo-ocular reflex during combined linear and angular acceleration. Exp Brain Res 1997;114:304-320.

Dai M, Klein A, Cohen B, Raphan T: Modelbased study of the human cupular time constant. J Vestib Res 1999;9:293-301.

Diamond S, Markham C: Validating the hypothesis of otolith asymmetry as a cause of space motion sickness. Ann NY Acad Sci 1992;656: 725-731.

Diamond S, Markham C: The effect of space missions on gravity-responsive torsional eye movements. J Vestib Res 1998;8:217-231.

-Furman JMR, Hain TC, Paige GD: Central adaptation models of the vestibulo-ocular and optokinetic systems. Biological Cybernetics 1989;61:255-264.

-Kingma H, Gullikers H, de Jong I, Jongen R, Dolmans M, Stegeman P: Real time binocular detection of horizontal, vertical and torsional eye movements by an infrared video-eye tracker. Acta Otolaryngol Suppl 1995;520: 9-15.

-Kingma H, Wuyts FL, Boumans L: Clinical testing of the statolith system in patients with Ménière's disease. Acta Otolaryngol Suppl 1997;526:24-26.

Kondrachuk AV: Qualitative model of otolithocular asymmetry in vertical eccentric rotation experiments. Hearing Res 2003;178:5969.

Levenberg K: A method for the solution of certain nonlinear problems in least squares. Q Appl Math 1944;4:164-168.
Malcolm R, Melville-Jones G: A quantitative study of vestibular adaptation in humans. Acta Otolaryngol 1970;70:126-135.

Marquardt DW: An algorithm for least-squares estimation of nonlinear parameters. J Soc Ind Appl Math 1963;11:431-441.

Melville-Jones G: Transfer function of labyrinthine volleys through the vestibular nuclei in Brodal A, Pompeiano O (eds): Basic Aspects of Central Vestibular Mechanisms. Amsterdam, Elsevier, 1972, pp 139-156.

Neuhauser HK, von Brevern M, Radtke A, Lezius F, Feldmann M, Ziese T, Lempert T: Epidemiology of vestibular vertigo: a neurotologic survey of the general population. Neurology 2005;65:898-904

Nooij SAE: Vestibular adaptation to an altered gravitational environment; thesis, Amersfoort, 2008.

Nowé V, Wuyts FL, Hoppenbrouwers M, van de Heyning PH, de Schepper AM, Parizel PM: The interutricular distance determined from external landmarks. J Vestib Res 2003; 13:17-23.

Oman CM, Young LR: Physiological range of pressure difference and cupula deflections in the human semicircular canal: theoretical considerations; in Brodal A, Pompeiano O (eds): Basic Aspects of Central Vestibular Mechanisms. Amsterdam, Elsevier, 1972, pp 529-539.

-Paige GD, Telford L, Seidman SH, Barnes GR: Human vestibule-ocular reflex and its interactions with vision and fixation distance during linear and angular head movement. J Neurophysiol 1998;80:2391-2404.

- Peterka RJ: Torsional vestibulo-ocular reflex measurements for identifying otolith asymmetries possibly related to space motion sickness susceptibility. Acta Astronaut 1994; 31:1-9.

-Raphan T, Matsuo V, Cohen B: Velocity storage in the vestibulo-ocular reflex arc (VOR). Exp Brain Res 1979;35:229-248.

Robinson DA: The use of control system analysis in the neurophysiology of eye movements. Ann Rev Neurosci 1981;4:463-504.

Smith ST, Curthoys IS, Moore ST: The human ocular torsion position response during yaw angular acceleration. Vision Res 1995;35: 2045-2055.
- Tarnutzer AA, Bockisch CJ, Straumann D: Head-roll-dependent variability of subjective visual-vertical and ocular counterroll. Exp Brain Res 2009;4:621-626.

Uchino Y, Sato H, Kushiro K, Zakir M, Imagawa M, Ogawa Y, Katsuta M, Isu N: Cross-striolar and commissural inhibition in the otolith system; in Cohan B, Hess BJM (eds): Otolith Function in Spatial Orientation and Movement. Annals of the New York Academy of Sciences. New York, New York Academy of Sciences, 1999, vol 871, pp 162-172.

von Baumgarten R, Thümler RA: A model for vestibular function in altered gravitational states. Life Sci Space Res 1979;17:626-632.

$\checkmark$ Wall C 3rd: The sinusoidal harmonic acceleration rotary chair test: theoretical and clinical basis. Neurol Clin 1990;8:269-285.

-Wetzig J, Reiser M, Martin E, Bregenzer N, von Baumgarten RJ: Unilateral centrifugation of the otoliths as a new method to determine bilateral asymmetries of the otolith apparatus in man. Acta Astronaut 1990;21:519-525.

Wilson VJ, Melville-Jones G: Mammalian Vestibular Physiology. New York, Plenum, 1979.

Wolfe JW, Engelken EJ, Kos CM: Low-frequency harmonic acceleration as a test of labyrinthine function: basic methods and illustrative cases. Otolaryngology 1978;86:130-142.

Wuyts FL, Furman JM, van de Heyning PH: Instrumentation and principles of vestibular testing; in Luxon L, et al (eds): Textbook of Audiological Medicine. Clinical Aspects of Hearing and Balance. London, Taylor and Francis, 2001, pp 717-734.

-Wuyts FL, Hoppenbrouwers M, Pauwels G, van de Heyning PH: Utricular sensitivity and preponderance assessed by the unilateral centrifugation test. J Vestib Res 2003;13:227234.

-Wuyts FL, Furman J, Vanspauwen R, van de Heyning PH: Vestibular function testing. Curr Opin Neurol 2007;20:19-24.

Yegorov BB, Samarin GI: Possible changes in the paired operation of the vestibular apparatus during weightlessness. Kosm Biol Aviakosm Med 1970;4:85-86 